\title{
Growth-inhibiting conditions slow growth plate senescence
}

\author{
Patricia Forcinito, Anenisia C Andrade ${ }^{1}$, Gabriela P Finkielstain, Jeffrey Baron, Ola Nilsson ${ }^{1,+}$ \\ and Julian C Lui ${ }^{\dagger}$
}

Developmental Endocrinology Branch, Program in Developmental Endocrinology and Genetics, Eunice Kennedy Shriver National Institute of Child Health and Human Development, National Institutes of Health, CRC, Room 1-3330, 10 Center Drive, MSC-1103, Bethesda, Maryland 20892-1103, USA

${ }^{1}$ Department of Women's and Children's Health, Center for Molecular Medicine and Pediatric Endocrinology Unit, Karolinska Institutet and Karolinska University Hospital, SE-171 76 Stockholm, Sweden

(Correspondence should be addressed to J C Lui; Email: luichunk@mail.nih.gov)

† ( $\mathrm{C}$ Lui and O Nilsson are the senior authors)

\begin{abstract}
The mammalian growth plate undergoes programed senescence during juvenile life, causing skeletal growth to slow with age. We previously found that hypothyroidism in rats slowed both growth plate chondrocyte proliferation and growth plate senescence, suggesting that senescence is not dependent on age per se but rather on chondrocyte proliferation. However, one alternative explanation is that the observed slowing of growth plate senescence is a specific consequence of hypothyroidism. We reasoned that, if delayed senescence is a general consequence of growth inhibition, rather than a specific result of hypothyroidism, then senescence would also be slowed by other growth-inhibiting conditions. In this study, we therefore used tryptophan
\end{abstract}

deficiency to temporarily inhibit growth in newborn rats for 4 weeks. We then allowed the animals to recover and studied the effects on growth plate senescence. We found that structural, functional, and molecular markers of growth plate senescence were delayed by prior tryptophan deficiency, indicating that the developmental program of senescence had occurred more slowly during the period of growth inhibition. Taken together with previous studies in hypothyroid rats, our findings support the hypothesis that delayed senescence is a general consequence of growth inhibition and hence that growth plate senescence is not simply a function of time per se but rather depends on growth.

Journal of Endocrinology (2011) 208, 59-67

\section{Introduction}

Mammalian body length is primarily determined by longitudinal bone growth that occurs at the growth plate, which is a thin layer of cartilage found near the ends of long bones. The process of longitudinal bone growth occurs by endochondral ossification, in which cartilage is formed and then remodeled into bone tissue (Kronenberg 2003). The growth plate is spatially organized into three layers: the resting zone, the proliferative zone, and the hypertrophic zone (Schenk \& Hunziker 1991). The resting zone chondrocytes are stem-like cells capable of generating new clones of proliferative chondrocytes (Abad et al. 2002). The proliferative zone and hypertrophic zone contain clones of chondrocytes, arranged in columns parallel to the long axis of the bone. The proliferative chondrocytes have a high rate of replication, and when they get farther from the epiphysis they stop dividing and become hypertrophic chondrocytes (Walker \& Kember 1972). Hypertrophic chondrocytes later undergo apoptosis, and the hypertrophic cartilage is invaded by blood vessels, osteoclasts, and differentiating osteoblasts, which remodel the cartilage into bone tissue (Kronenberg 2003).
Longitudinal bone growth is rapid in early life but gradually slows with age, until growth velocity eventually approaches zero in adulthood. The decline in longitudinal bone growth with age is associated with functional, structural, and molecular changes in the growth plate known as growth plate senescence (Nilsson \& Baron 2004). These changes include a decline in the chondrocyte proliferation rate (Walker \& Kember 1972), overall growth plate height, proliferative and hypertrophic zone heights, column density, and extensive changes in gene expression (Gafni et al. 2001, Schrier et al. 2006, Marino et al. 2008, Lui et al. 2010a).

The physiological process that drives growth plate senescence is not known. One possibility is that senescence is a function of time per se, implying the existence of a biological clock in the growth plate. Another possibility is that senescence is actually a function of growth. For example, growth plate chondrocytes might have a finite proliferative capacity, which is gradually exhausted, leading to the decline in growth rate and other senescent changes in the growth plate. The latter hypothesis that senescence is a function of growth can be tested by investigating whether inhibition of longitudinal bone growth affects growth plate senescence. If senescence were a function of growth, then growth 
inhibition would be expected to slow senescence; if senescence were simply a function of time, then growth inhibition would not slow senescence. We previously showed that inhibiting bone growth in newborn rats by inducing hypothyroidism slowed both growth plate chondrocyte proliferation as well as growth plate senescence, suggesting that senescence is driven by growth (Marino et al. 2008). However, one alternative explanation for these findings is that the observed slowing of growth plate senescence is a specific consequence of hypothyroidism, which exerts complex and widespread effects on growth plate cartilage, rather than a general consequence of growth inhibition.

The goal of this study is to determine whether delayed growth plate senescence is a specific consequence of hypothyroidism or a more general consequence of growth inhibition. We therefore used another growth-inhibiting condition, tryptophan deficiency, to temporarily suppress growth in newborn rats for 4 weeks. Afterwards, growth in these rats was allowed to recover by switching to a replete diet. These rats were then studied to determine whether the prior period of growth inhibition had slowed growth plate senescence by examining structural, functional, and molecular markers of growth plate senescence.

\section{Materials and Methods}

\section{Animal care}

All animal procedures were approved by the National Institute of Child Health and Human Development Animal Care and Use Committee (National Research Council 2003).

\section{Animal procedures for normal development assessment}

Sprague-Dawley rats (Harlan, Indianapolis, IN, USA) received standard rodent chow (Zeigler Bros, Gardners, PA, USA) and water, made available ad libitum. Male rats (six per time point) were killed by carbon dioxide inhalation at ages 1 , $3,6,9$, and 12 weeks. To study the growth plate without the complicating effects of sex steroids, 3-, 6-, 9-, and 12-weekold rats were castrated at 18 days of age. Proximal tibial growth plates were excised, embedded in optimum cutting temperature (OCT) compound (Electron Microscopy Sciences, Hatfield, PA, USA), and stored at $-80{ }^{\circ} \mathrm{C}$ until subsequent microdissection.

\section{Animal procedures for growth inhibition by tryptophan deficiency}

Sprague-Dawley rats were obtained from Charles River Laboratories (Wilmington, MA, USA) and provided with food and water ad libitum. Growth was inhibited by providing a tryptophan deficient $\left(\operatorname{Trp}^{-}\right)$diet to the lactating mother from birth of the male pups until they reached 4 weeks of age as previously described (Lui et al. 2010b). Male pups of lactating mothers receiving a diet with adequate tryptophan for normal nutritional needs were used as controls. At 4 weeks of age, all animals were switched to regular chow. Control and treated animals were killed at 1, 3, 4, 5, 6, 8, 12, 16, and 20 weeks of age and tibial length was measured using a digital caliper. From each animal, one proximal tibial growth plate was excised, embedded in OCT compound, and stored at $-80^{\circ} \mathrm{C}$ until subsequent microdissection and the other was fixed in formalin for quantitative histology.

\section{Animal procedures for growth inhibition by hypothyroidism}

Sprague-Dawley rats were obtained from Harlan Laboratories and provided with food and water ad libitum. Growth was inhibited in newborn male pups by introducing propylthiouracil (PTU, supplied by Sigma-Aldrich) into the drinking water of the mother at a concentration $1 \mathrm{~g} / \mathrm{l}$ beginning when the pups were 1 day of age as previously described (Marino et al. 2008). PTU treatment was discontinued at 5 weeks of age. The PTU-treated animals were not weaned during the experiment because preliminary studies showed that they were not mature enough to support their own nutritional needs. Pups from lactating mothers not receiving PTU were used as controls and weaned at the age of 3 weeks. All rats were given depot leuprolide acetate $(6 \mathrm{mg} / \mathrm{kg}$, s.c.; Tap Pharmaceuticals, Deerfield, IL, USA) every 3 weeks, starting at 3 weeks of age. Leuprolide acetate is a long-acting GnRH agonist that downregulates gonadotropin secretion and thus gonadal steroid production (Ogawa et al. 1989). Control and PTU-treated animals were killed and studied after the end of the treatment period, at 7 and 9 weeks of age $(n=6$, each age group). The proximal tibiae growth plate was excised and frozen in dry ice, stored at $-80^{\circ} \mathrm{C}$ until subsequent microdissection.

\section{Growth plate microdissection, RNA extraction}

Growth plate microdissection was performed as previously described (Nilsson et al. 2007). Briefly, $50 \mu \mathrm{m}$ frozen sections were thawed, fixed in ethanol, stained with eosin, dehydrated, and placed in xylene. Using an inverted microscope, the proliferative plus early hypertrophic zone was collected by manual microdissection. RNA (100-150 ng) was extracted from growth plate samples as previously described (Nilsson et al. 2007). RNA quality was assessed using an Agilent 2100 Bioanalyzer (Agilent Technologies, Santa Clara, CA, USA).

\section{Quantitative real-time PCR}

RNA was reverse transcribed using Superscript III reverse transcriptase (Invitrogen) following the manufacturer's instructions. Quantitative real-time PCR was performed using commercially available 6-carboxyfluorescein (FAM) or VIC-labeled Taqman assays, or custom-designed primers coupled with SYBR green real-time PCR reagent (Applied Biosystems, Foster City, CA, USA). The assay ID or sequence 
information for different assays and primers are as follow: 18S, 4319413E; insulin-like growth factor-binding protein 7 (Igfbp7), Rn01413246_m1; HtrA serine peptidase 1 (Prss11), Rn00581870_m1; PYD and CARD domain containing (Pycard), Rn00597229_g1; retinoid X receptor, gamma $(R x R g)$, Rn01483466_m1; Igf2, Rn01454518_m1; calcitonin (Calca), Trp ${ }^{-}$experiment: Rn01511353_g1, hypothyroidism experiment: Rn00569199_m1; cyclindependent kinase inhibitor $2 \mathrm{~A}(C d k n 2 a), \operatorname{Trp}{ }^{-}$experiment: Rn00580664_m1, hypothyroidism experiment: forward primer 5'-GGGTCACCGACAGGCATAAC, reverse primer 5'-GCCTAACTTA GCGCTGCTTTG; ankyrinrepeat and suppressor of cytokine signaling box-containing protein 4 (Asb4), Trp ${ }^{-}$experiment: Rn01501048_m1, hypothyroidism experiment: forward primer $5^{\prime}-\mathrm{TC}-$ GTCTGTGCCAAGCAGTTG, reverse primer $5^{\prime}$-CCTGGTTGTTGGTTTTCATGTTC. Reactions were performed in triplicate on cDNA derived from individual animals using the ABI prism 7900 Sequence Detection System instrument (Applied Biosystems). The relative quantity of each mRNA was calculated using the formula: relative expression $=2^{-\Delta C_{\mathrm{t}}} \times 10^{9}$, where $C_{\mathrm{t}}$ represents the threshold cycle and $\Delta C_{\mathrm{t}}=\left(C_{\mathrm{t}}\right.$ of gene of interest $)-\left(C_{\mathrm{t}}\right.$ of $18 \mathrm{~S}$ rRNA). Values were multiplied by $10^{9}$ for convenience of comparison. For the comparison between $\operatorname{Trp}^{-}$animals and controls, three to five animals were studied per treatment and time point. For the comparison between hypothyroid animals and controls, six animals were studied per treatment and time point.

\section{Quantitative histology}

Growth plate samples fixed in formalin were decalcified in 0.5 M pH 7.4 EDTA (KD Medical, Columbia, MD, USA), embedded in paraffin, cut into $10 \mu \mathrm{m}$ sections, and stained with Masson's trichrome stain. ScanScope CS digital scanner (Aperio Technologies, Inc., Vista, CA, USA) was used to visualize and analyze the stained sections. Measurements were taken as previously described (Marino et al. 2008) with some modifications. The following measurements were made by an observer blinded to the age and treatment group: overall growth plate height, measured from the margin of the metaphyseal bone to the margin of the epiphyseal bone; resting zone height, measured from the edge of the margin of the epiphyseal bone to the first cell of a proliferative column; hypertrophic zone height, measured from the margin of the methaphyseal bone to the last cell bigger or equal to $10 \mu \mathrm{m}$; proliferative zone height, measured from the first cell minor to $10 \mu \mathrm{m}$ to the first resting chondrocytes; the number of resting zone chondrocytes, assessed as the number of cells per $200 \mu \mathrm{m}$ growth plate width (measured parallel to the epiphyseal margin); column density, assessed as the number of proliferative columns per $500 \mu \mathrm{m}$ growth plate (measured parallel to the metaphyseal margin); and the height of the terminal hypertrophic chondrocyte lacuna (the intact lacuna closest to the metaphyseal bone). All histological measurements were performed in the central two-thirds of the growth plate sections for avoiding regions close to the perichondrium. Heights were measured parallel to the chondrocyte columns in three areas of each growth plate section and averaged. Column density was calculated in two areas per growth plate section. The number of proliferative and hypertrophic cells was counted in three to four intact columns per growth plate. Hypertrophic chondrocytes were operationally defined by a height $\geq 10 \mu \mathrm{m}$. The terminal hypertrophic cell height was measured in eight to ten different columns per growth plate section. For each animal, three different growth plate sections were analyzed and then all measurements from that animal were averaged. Four to five animals were studied per treatment and time point.

\section{Statistical analysis}

The SigmaStat 3.1 statistical program was used to perform all statistical measurements. One-way ANOVA was used to assess the effect of age during normal development and in untreated controls. Two-way ANOVA was used to assess the effect of prior treatment in the hypothyroidism and tryptophan-deficient experiments. For these analyses, we analyzed time points after the end of the treatment period using time and prior treatment as independent variables. All real-time PCR data were log transformed prior to statistical testing.

\section{Results}

Tryptophan deficiency delayed structural and functional senescent changes in the growth plate

Early postnatal growth was delayed in rats from birth until 4 weeks of age by providing a $\operatorname{Trp}^{-}$diet to the lactating mother. At the end of the 4-week treatment period, the tibial length was $16.5 \pm 0.4 \mathrm{~mm}$ in $\operatorname{Trp}^{-}$rats versus 26.6 $\pm 0.2 \mathrm{~mm}$ in control rats fed with a replete diet $(P<0 \cdot 001)$. The rats were then placed on normal chow, allowed to recover, and then assessed to determine whether the prior period of tryptophan deficiency and growth inhibition had delayed growth plate senescence. In control animals, many structural features of the growth plate changed significantly $(P<0 \cdot 05)$ with age (Fig. 1, solid lines), including the growth plate height, the proliferative zone height, the number of proliferative chondrocytes per column, and the number of resting zone chondrocytes per $200 \mu \mathrm{m}$. Other structural features (such as resting zone height), however, did not show significant changes, which might be due to the limited age range (4-8 weeks) of this study. In animals that had previously been tryptophan deficient, most of these changes were significantly delayed compared to controls (Fig. 1A) except for the number of hypertrophic zone chondrocytes per column $(P=0.053)$ and column 

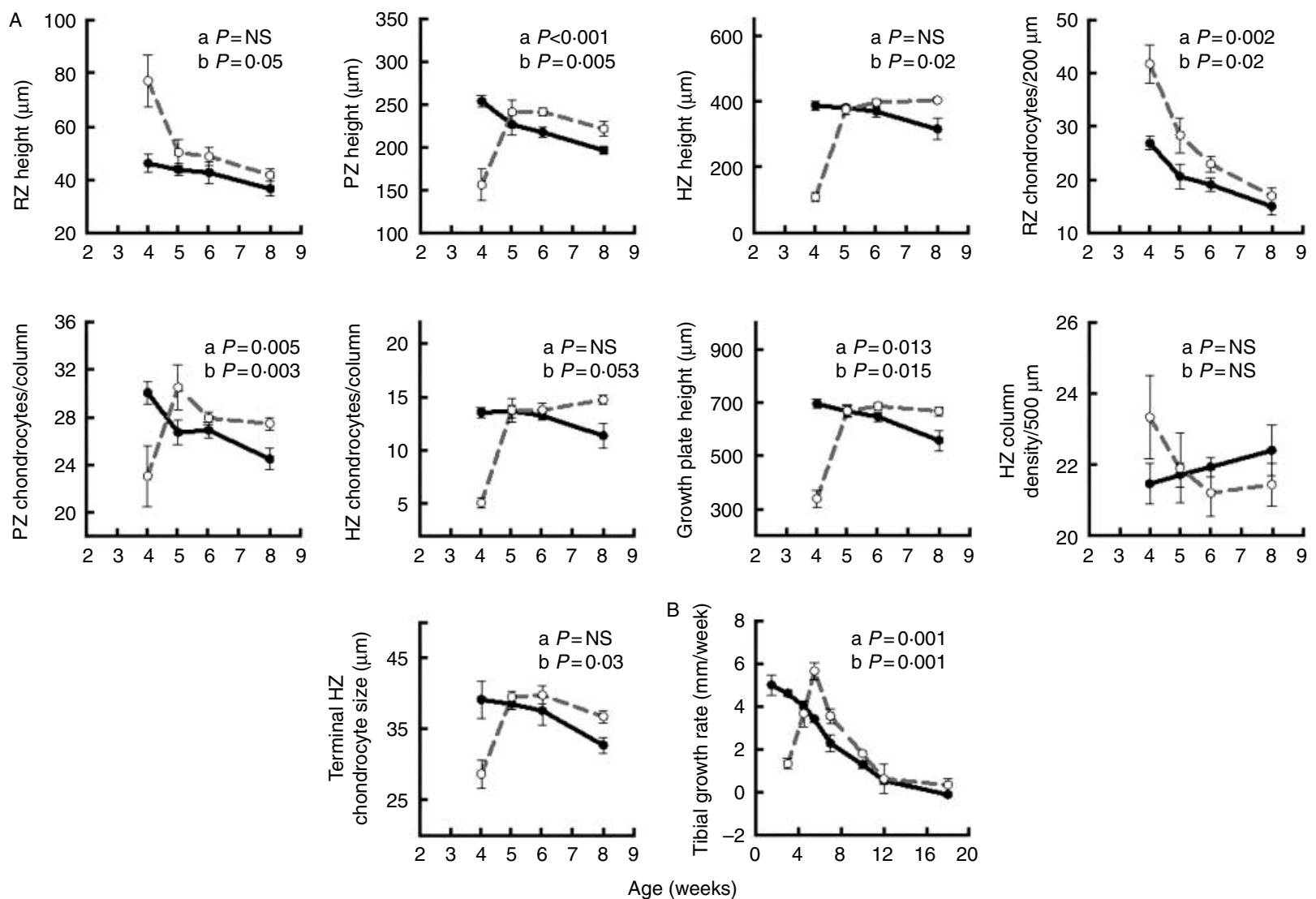

Figure 1 Structural and functional senescent changes in the growth plate were delayed by prior tryptophan deficiency. Markers of growth plate senescence were compared in rat proximal tibia's growth plate Masson's trichrome-stained sections for control or untreated rats (solid lines) and rats that received a $\operatorname{Trp}^{-}$diet from birth to 4 weeks of age (dotted line). Between 4 and 5 weeks of age, the Trp ${ }^{-}$rats were going through a transition between being growth inhibited and resuming normal growth. (A) Structural markers were measured at different ages, including resting, proliferative, and hypertrophic zones height; number of resting zone chondrocytes per $200 \mu \mathrm{m}$ of growth plate width; number of proliferative and hypertrophic chondrocytes per column; growth plate height; number of hypertrophic columns or column density per $500 \mu \mathrm{m}$ of growth plate width; and terminal hypertrophic chondrocytes height. Each experimental group contained four to five animals. (B) Rate of proximal tibia growth per week, defined as the increase in proximal tibial height from the previous time point divided by the number of weeks between the two time points. a, effect of age, one-way ANOVA; b, effect of treatment during the recovery period, two-way ANOVA. RZ, resting zone; PZ, proliferative zone; $\mathrm{HZ}$, hypertrophic zone; NS, not significant.

density. The delay in structural changes also was evident by visual inspection of the growth plate sections (Fig. 2). For example, the overall growth plate height during tryptophan deficiency (at 4 weeks of age) was much shorter than in controls, but after the tryptophan deficiency resolved (at 6-8 weeks of age), the growth plate height exceeded the height in control animals. Similarly, the tibial growth rate, which is a functional marker of growth plate senescence, declined in control animals $(P<0 \cdot 001$; Fig. 1B) and was delayed by prior tryptophan deficiency $(P<0 \cdot 001$; Fig. 1B).

\section{Tryptophan deficiency delayed senescence-associated changes in gene expression}

We next asked whether tryptophan deficiency also delayed senescence-associated changes in gene expression. We recently reported that hundreds of genes show significant changes in gene expression with postnatal age in the rat proliferative zone plus early hypertrophic zone, as assessed by expression microarray analysis (Lui et al. 2010a). Based on that expression data (GEO accession number GSE16981), we focused on five genes that showed increasing expression with age (Table 1): $\operatorname{Igfbp} 7$; Pycard, which is involved in apoptotic signaling pathways; $C d k n 2 a$, a tumor suppressor gene; Prss11, which regulates availability of IGFs; and Calca, which regulates extracellular calcium. We also studied three molecular markers that decrease their expression during senescence (Table 1), including $I g f 2 ; A s b 4$; and $R x R g$, which modulates the actions of multiple nuclear receptors. Firstly, we used real-time PCR to verify the microarray findings. In the control animals, real-time PCR confirmed that all these genes showed significant $(P<0 \cdot 05)$ changes in expression with age (Fig. 3, left column of graphs). We then asked whether the animals that were previously $\operatorname{Trp}^{-}$but had then 


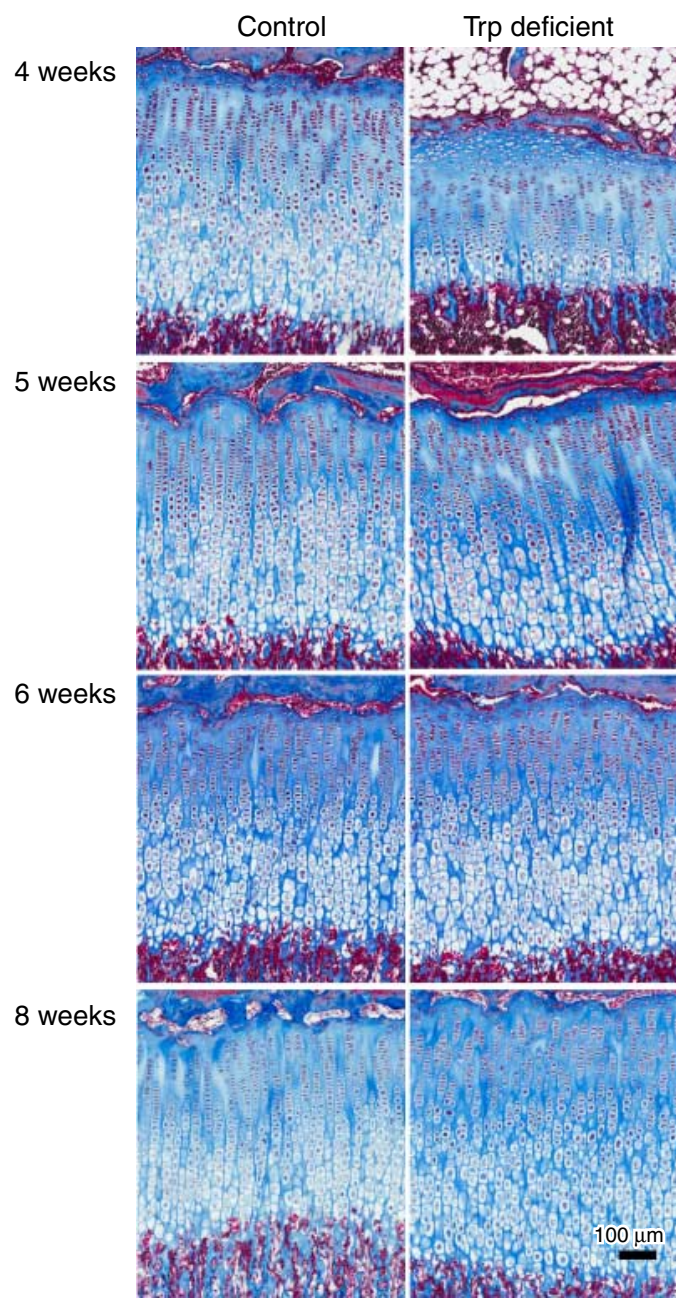

Figure 2 Growth plate structure in 4-, 5-, 6-, and 8-week-old control and $\operatorname{Trp}^{-}$animals. Photomicrographs of Masson's trichrome-stained longitudinal sections of proximal tibial growth plate from control and $\operatorname{Trp}^{-}$animals at different ages. During the period of growth inhibition (4 weeks), the overall growth plate height in the $\mathrm{Trp}^{-}$animals was smaller than the control animals, despite having significantly more resting chondrocytes. At $\sim 5$ weeks of age, the experimental group was in the process of recovering from the growth inhibition (see Fig. 1B), which may explain why the growth plate height media was similar to that of control animals. Afterwards, at 6 and 8 weeks of age, the growth plate height was greater in animals that had previously received a $\operatorname{Trp}^{-}$diet than in control animals. Each experimental group contained four to five animals. Bar represents $100 \mu \mathrm{m}$. Full colour version of this figure available via http://dx.doi.org/10.1677/ JOE-10-0302.

resumed normal growth showed a delay in the age-dependent changes in gene expression compared to controls. At 5-8 weeks of age, the expression of Igfbp 7, Calca, Prss 11, and Pycard was significantly $(P<0 \cdot 05)$ lower in the previously Trp $^{-}$animals than in controls. As the expression of these genes normally rises during senescence, the findings indicate that prior tryptophan deficiency delayed the normal increase
(Fig. 3A, middle column of graphs). Conversely, for Igf2, prior tryptophan deficiency delayed the normal decrease in expression (Fig. 3B, middle column of graphs). For Cdkn2a, $A s b 4$, and $R \times R g$, gene expression did not differ significantly between control and treated animals during the recovery period. However, for these three genes, the changes in expression in the control group were modest during the time period studied. Therefore, a delayed change in expression in the experimental group might be more difficult to detect.

Growth inhibition due to hypothyroidism also delayed senescenceassociated changes in gene expression

We then used a second model of growth inhibition and assessed the effects on the same set of genes. Newborn rats were rendered hypothyroid by addition of PTU to the drinking water. We previously demonstrated that this intervention does cause hypothyroidism, growth inhibition, and delay of structural and functional markers of growth plate senescence (Marino et al. 2008). In this study, we shortened the duration of the PTU treatment from 0 to 8 weeks to 0 to 5 weeks, and studied the effects of gene expression. mRNA levels were measured by real-time PCR at 7 and 9 weeks of age, that is after the removal of PTU from drinking water at 5 weeks. The normal senescence-associated changes in expression were delayed significantly $(P<0 \cdot 05)$ by prior hypothyroidism for Igfbp 7, Prss 11, Calca, Pycard, Igf2, and Asb4. Thus, hypothyroidism, similar to tryptophan deficiency, caused a delay in most of the senescence-associated genes studied. Expression of $C d k n 2 a$ and $R \times R g$ was not significantly different in control versus treated animals. However, for these two genes, there was mininal change in expression in the control group during the time period studied, which might make a delayed change in expression more difficult to detect.

\section{Discussion}

We previously showed that hypothyroidism slows both growth plate chondrocyte proliferation and growth plate senescence in newborn rats (Marino et al. 2008), suggesting that growth plate senescence is a function of growth. However, one alternative explanation is that the observed effect on growth plate senescence is a specific consequence of hypothyroidism, which exerts complex and widespread effects on growth plate cartilage (Nilsson et al. 2005a), including direct effects on proliferation and hypertrophy (Stevens et al. 2000), and indirect effects mediated by decreased GH (Martin et al. 1985, Katakami et al. 1986) and IGF1 (Kindblom et al. 2001). If delayed growth plate senescence is a general consequence of growth inhibition, rather than a specific consequence of hypothyroidism, then senescence would also be delayed by other growth-inhibiting conditions. We therefore used a second model of growth inhibition in this study by inducing tryptophan deficiency in newborn rats. We then allowed the animals to recover and studied the effects on growth 
Table 1 Changes in gene expression during senescence by Affymetrix Rat Genome Array 2302.0

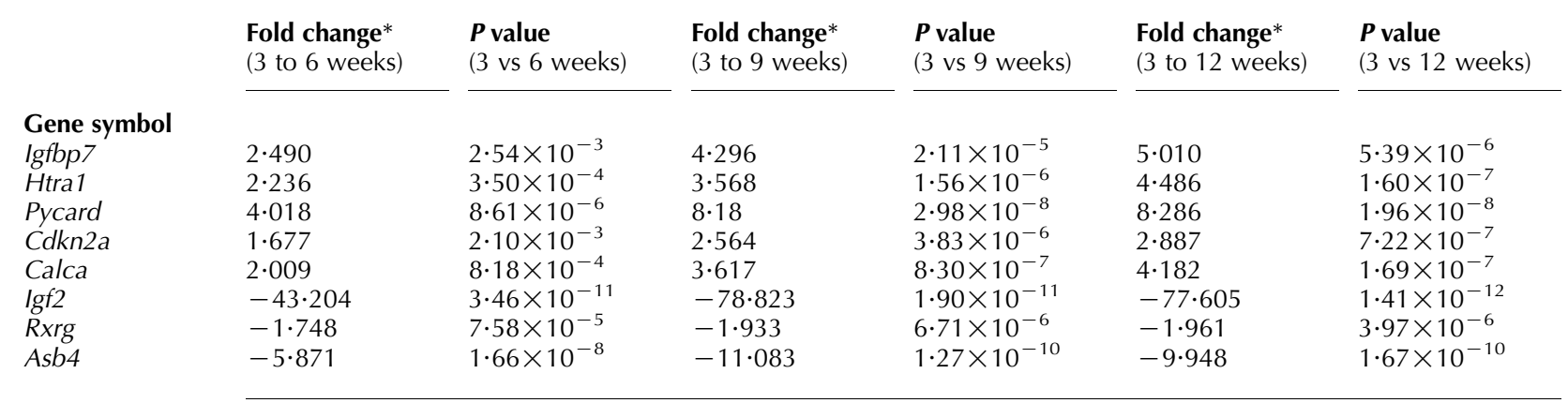

*Negative fold changes indicate declining expression with age.

plate senescence. We found that structural, functional, and molecular markers of growth plate senescence were delayed by prior tryptophan deficiency, indicating that the developmental program of senescence had occurred more slowly during the period of growth inhibition. Taken together, our findings in the hypothyroidism model and in the tryptophandeficient model support the hypothesis that delayed senescence is a general consequence of growth inhibition.

The tryptophan-deficient model that we chose in this study may inhibit bone growth through multiple mechanisms. Firstly, it has been shown that single amino acid restriction may inhibit the nutrient-sensing mechanistic target of rapamycin pathway in the growth plate (Kim et al. 2009), which inhibits chondrocyte proliferation and differentiation (Phornphutkul et al. 2008, 2009, Kim et al. 2009). Secondly, tryptophan deficiency causes depletion of brain serotonin (Biggio et al. 1974) and thereby diminishes appetite and food intake, causing malnutrition (D'Souza et al. 2004). This generalized malnutrition could in turn have systemic effects on the growth plate, in part through diminished GH and IGF1 levels (Heinrichs et al. 1997, Gat-Yablonski et al. 2009). Hypothyroidism has a direct effect on growth plate but can also decrease GH and IGF1 levels (Nilsson et al. 2005a). Therefore, there may be some overlap in the mechanisms by which tryptophan deficiency and hypothyroidism affect the growth plate and thus the two models may not be completely independent. However, the fact that both tryptophan deficiency and hypothyroidism, as well as a third model using dexamethasone excess in rabbit, though with more limited data (Baron et al. 1994, Gafni et al. 2001), all showed delayed growth plate senescence, strongly support the hypothesis that delayed growth plate senescence is a general consequence of growth inhibition, which in turn suggests that growth plate senescence is not simply a function of time per se but rather depends on growth.

The underlying cellular mechanisms by which growth may drive senescence remain to be elucidated. Several lines of argument suggest that growth plate senescence may be dependent on the proliferative history of the resting zone chondrocytes. Firstly, the resting chondrocyte appears to be the only long-term cellular resident in the growth plate because proliferative chondrocytes differentiate into hypertrophic chondrocytes, which then undergo apoptosis (Kronenberg 2003). Therefore, the resting chondrocyte is a good candidate to store long-term information regarding the growth history of the growth plate. Secondly, the resting zone chondrocytes appear to act as a stem-like cell in the postnatal growth plate, capable of generating new clones of proliferative and hypertrophic chondrocytes (Abad et al. 2002). Therefore, senescence-associated changes in the resting chondrocyte could affect its proliferative and hypertrophic cellular progeny, thus accounting for the widespread changes characteristic of growth plate senencences. Thirdly, with age, there is a gradual decline in the number of resting chondrocytes as well as in resting chondrocyte proliferation, which may contribute to growth plate senescence (Schrier et al. 2006). Thus, resting zone chondrocytes may have a finite proliferative capacity, which is gradually exhausted. Consequently, growthinhibiting conditions, by slowing resting zone chondrocyte proliferation, may preserve the future proliferative potential of these progenitor cells and thereby slow senescence.

Similarly, the molecular mechanism by which growth may advance the senescence program is unknown. One possibility is that proliferation leads to alterations in epigenetic marks, which are known to participate in cellular memory (Ringrose \& Paro 2004, Bantignies \& Cavalli 2006). Limited evidence suggests that the level of DNA methylation in the resting zone chondrocytes decreases with age (Nilsson et al. 2005b) but it is unclear whether this observation is causally related to their replicative potential. In other juvenile tissues, as somatic growth slows, there is a decline in histone H3K4 methylation in the promoter region of growth-promoting genes, which may lead to their downregulation (Lui et al. 2010b).

A postnatal decline in growth rate is observed not only in the long bones, but also in many other organs. It is yet unclear how growth in different parts of the body slows concordantly to maintain body proportion. In transplantation experiments, growth of the transplanted organs generally depends on the age of the donor, suggesting that the growth deceleration in other organs may involve a local mechanism similar to that of the growth plate (Cooke et al. 1986, Pape et al. 2006). Interestingly, recent studies suggest that growth deceleration 
in the liver, kidney, lung, and heart depends on a genetic program involving the downregulation of multiple growthpromoting genes and suggest that this program can be delayed by prior growth inhibition (Lui et al. 2008, 2010b, Finkielstain

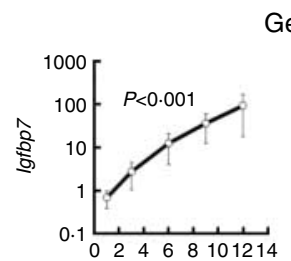

Genes upregulated with age
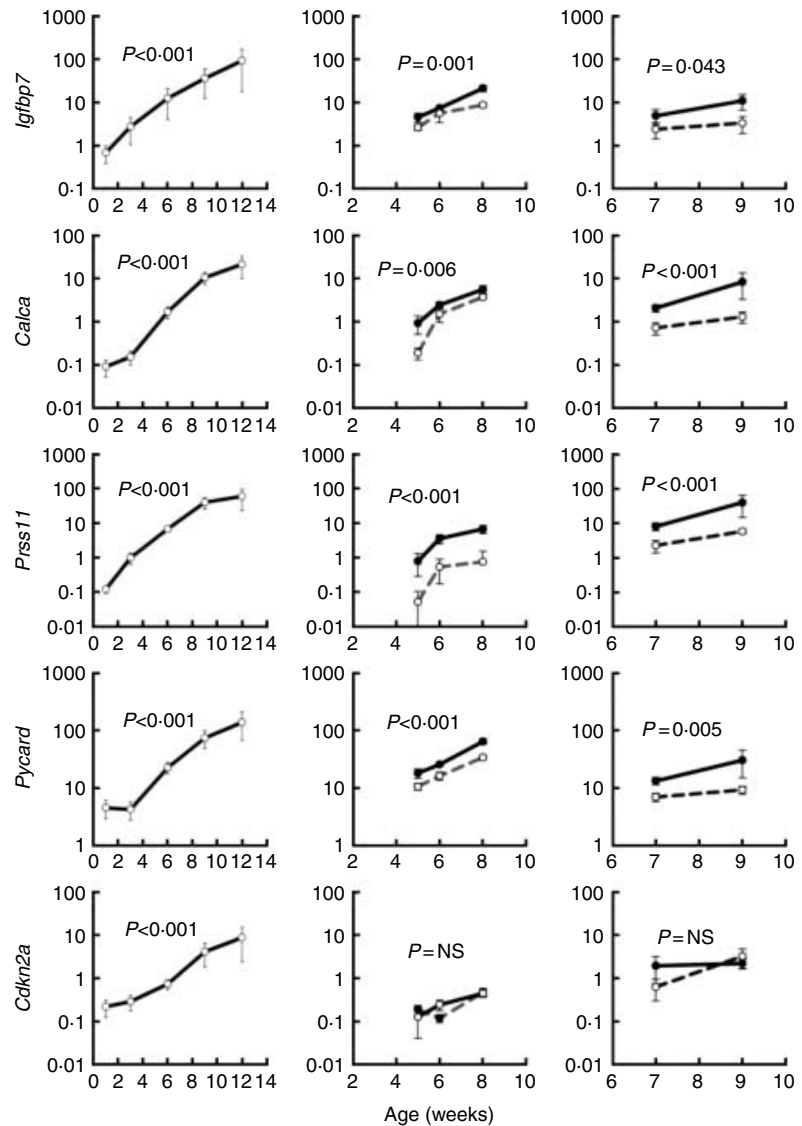

Genes downregulated with age
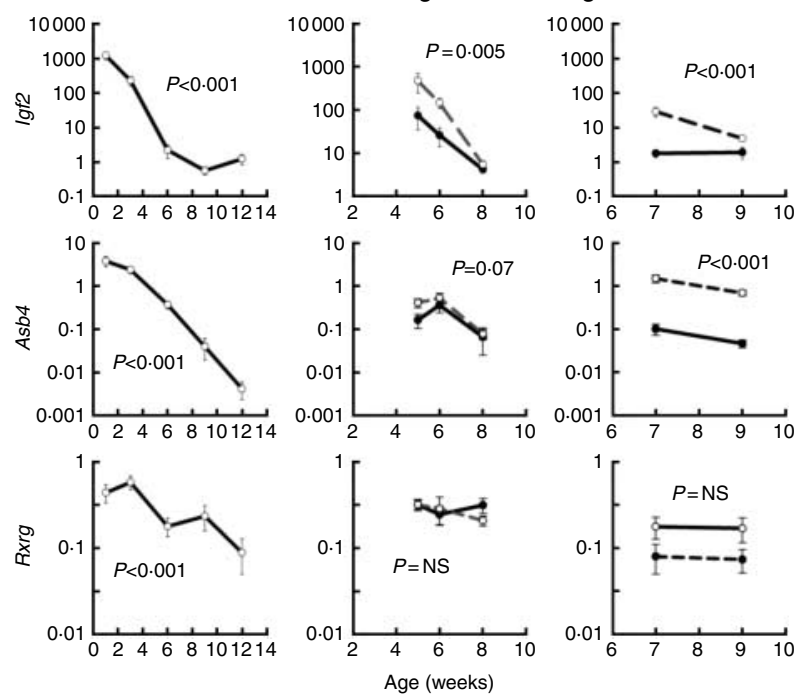

et al. 2009). Thus, the mechanism slowing growth of nonskeletal tissues also appears to be driven by growth, rather than by time alone. It will be intriguing to determine whether the two processes - growth plate senescence and growth deceleration in the soft tissue - is governed by similar mechanisms, and if certain types of cells in the soft tissue may function as 'stem-like' progenitor cells analogous to the resting zone chondrocytes in the growth plate.

Finally, our findings also provide important insights into the mechanism of catch-up growth. As previously suggested (Nilsson \& Baron 2004), delayed growth plate senescence may partly explain catch-up growth, which refers to accelerated growth above the normal rate for age after remission from growth inhibitory conditions. It is yet unclear whether catch-up growth after different types of growth inhibition is explained by delayed growth plate senescence. This hypothesis has previously been studied in animal models involving hypothyroidism and glucocorticoid excess to inhibit growth (Gafni et al. 2001, Marino et al. 2008). One clinical study has shown that the pattern of catch-up growth in children recovering from celiac disease is consistent with the hypothesis of delayed growth plate senescence (Emons et al. 2005). In this study, we found that animals previously receiving a $\operatorname{Trp}^{-}$diet had tibial growth rates higher than the control animals (Fig. 1B). The Trp ${ }^{-}$animals appeared to have a delayed decline in tibial growth rate of $\sim 2$ weeks (Fig. 1B). Thus, for example, at 9 weeks of age, the rats exhibited a rate of longitudinal growth similar to that of a 7 -week-old control animal. Notably, this delay of $\sim 2$ weeks was similar in magnitude to the delay in structural and molecular markers of the growth plates. Therefore, our findings here provide strong support for the hypothesis that catch-up growth after malnutrition can be explained at least in part by a delay in the program of growth plate senescence. Taken together with previous findings that delayed growth plate senescence contributes to catch-up growth after hypothyroidism and glucocorticoid excess, our data suggest that delayed growth plate senescence is a general mechanism that contributes to catch-up growth after a variety of growth-inhibiting conditions.

In summary, we used tryptophan deficiency to temporarily inhibit growth in newborn rats from birth until 4 weeks of age. We then studied structural, functional, and molecular markers of growth plate senescence and found that they were delayed by prior tryptophan deficiency. This finding indicates that the developmental program of senescence had occurred more slowly during the period of growth inhibition.

Figure 3 Prior tryptophan deficiency or prior hypothyroidism delayed senescent changes of gene expression in the growth plate. mRNA levels were measured by real-time PCR at various ages in castrated male rats (left column) and in rats that had previously received a tryptophan-deficient diet (dotted line, middle column) or received PTU to induce hypothyroidism (dotted line, right column) along with corresponding controls (solid lines). Each experimental group contained three to six animals. NS, not significant. 
Taken together with previous studies in hypothyroid rats, our findings support the hypothesis that delayed senescence is a general consequence of growth inhibition, suggesting that growth plate senescence is not simply a function of time per se but rather depends on growth.

\section{Declaration of interest}

The authors declare that there is no conflict of interest that could be perceived as prejudicing the impartiality of the research reported.

\section{Funding}

This research was supported by the Intramural Research Program of the Eunice Kennedy Shriver National Institute of Child Health and Human Development, NIH. ON was supported by grants from the Swedish Research Council (K2007-52X-20316-01-4), the Swedish Society of Medicine, HKH kronprinsessan Lovisas Förening för Barnasjukvård, Sällskapet Barnavård, and Stiftelsen Frimurare Barnhuset i Stockholm.

\section{References}

Abad V, Meyers JL, Weise M, Gafni RI, Barnes KM, Nilsson O, Bacher JD \& Baron J 2002 The role of the resting zone in growth plate chondrogenesis. Endocrinology 143 1851-1857. (doi:10.1210/en.143.5.1851)

Bantignies F \& Cavalli G 2006 Cellular memory and dynamic regulation of polycomb group proteins. Current Opinion in Cell Biology 18 275-283. (doi:10.1016/j.ceb.2006.04.003)

Baron J, Klein KO, Colli MJ, Yanovski JA, Novosad JA, Bacher JD \& Cutler GB Jr 1994 Catch-up growth after glucocorticoid excess: a mechanism intrinsic to the growth plate. Endocrinology 135 1367-1371. (doi:10.1210/ en.135.4.1367)

Biggio G, Fadda F, Fanni P, Tagliamonte A \& Gessa GL 1974 Rapid depletion of serum tryptophan, brain tryptophan, serotonin and 5-hydroxyindoleacetic acid by a tryptophan-free diet. Life Sciences $\mathbf{1 4}$ 1321-1329. (doi:10.1016/0024-3205(74)90440-8)

Cooke PS, Yonemura CU, Russell SM \& Nicoll CS 1986 Growth and differentiation of fetal rat intestine transplants: dependence on insulin and growth hormone. Biology of the Neonate 49 211-218. (doi:10.1159/ $000242533)$

D'Souza DN, Zhang Y, Garcia F, Battaglia G \& Van de Kar LD 2004 Fluoxetine-induced changes in body weight and 5-HT1A receptormediated hormone secretion in rats on a tryptophan-deficient diet. American Journal of Physiology. Regulatory, Integrative and Comparative Physiology 286 R 390-R397. (doi:10.1152/ajpregu.00335.2003)

Emons JA, Boersma B, Baron J \& Wit JM 2005 Catch-up growth: testing the hypothesis of delayed growth plate senescence in humans. Journal of Pediatrics 147 843-846. (doi:10.1016/j.jpeds.2005.07.033)

Finkielstain GP, Forcinito P, Lui JC, Barnes KM, Marino R, Makaroun S, Nguyen V, Lazarus JE, Nilsson O \& Baron J 2009 An extensive genetic program occurring during postnatal growth in multiple tissues. Endocrinology 150 1791-1800. (doi:10.1210/en.2008-0868)

Gafni RI, Weise M, Robrecht DT, Meyers JL, Barnes KM, De-Levi S \& Baron J 2001 Catch-up growth is associated with delayed senescence of the growth plate in rabbits. Pediatric Research 50 618-623. (doi:10.1203/ 00006450-200111000-00014)

Gat-Yablonski G, Yackobovitch-Gavan M \& Phillip M 2009 Nutrition and bone growth in pediatrics. Endocrinology and Metabolism Clinics of North America 38 565-586. (doi:10.1016/j.ecl.2009.07.001)

Heinrichs C, Colli M, Yanovski JA, Laue L, Gerstl NA, Kramer AD, Uyeda JA \& Baron J 1997 Effects of fasting on the growth plate: systemic and local mechanisms. Endocrinology 138 5359-5365. (doi:10.1210/en.138.12.5359)
Katakami H, Downs TR \& Frohman LA 1986 Decreased hypothalamic growth hormone-releasing hormone content and pituitary responsivenes in hypothyroidism. Journal of Clinical Investigation 77 1704-1711. (doi:10. 1172/JCI112490)

Kim MS, Wu KY, Auyeung V, Chen Q, Gruppuso PA \& Phornphutkul C 2009 Leucine restriction inhibits chondrocyte proliferation and differentiation through mechanisms both dependent and independent of mTOR signaling. American Journal of Physiology. Endocrinology and Metabolism 296 E1374-E1382. (doi:10.1152/ajpendo.91018.2008)

Kindblom JM, Gothe S, Forrest D, Tornell J, Tornell J, Vennstrom B \& Ohlsson C $2001 \mathrm{GH}$ substitution reverses the growth phenotype but not the defective ossification in thyroid hormone receptor alpha $1-/-$ beta / - mice. Journal of Endocrinology 171 15-22. (doi:10.1677/joe.0.1710015)

Kronenberg HM 2003 Developmental regulation of the growth plate. Nature 423 332-336. (doi:10.1038/nature01657)

Lui JC, Finkielstain GP, Barnes KM \& Baron J 2008 An imprinted gene network that controls mammalian somatic growth is down-regulated during postnatal growth deceleration in multiple organs. American Journal of Physiology. Regulatory, Integrative and Comparative Physiology 295 R189-R196. (doi:10.1152/ajpregu.00182.2008)

Lui JC, Andrade AC, Forcinito P, Hegde A, Chen W, Baron J \& Nilsson O $2010 a$ Spatial and temporal regulation of gene expression in the mammalian growth plate. Bone 46 1380-1390. (doi:10.1016/j.bone.2010.01.373)

Lui JC, Forcinito P, Chang M, Chen W, Barnes KM \& Baron J $2010 b$ Coordinated postnatal down-regulation of multiple growth-promoting genes: evidence for a genetic program limiting organ growth. FASEB Journal 24 3083-3092. (doi:10.1096/fj.09-152835)

Marino R, Hegde A, Barnes KM, Schrier L, Emons JA, Nilsson O \& Baron J 2008 Catch-up growth after hypothyroidism is caused by delayed growth plate senescence. Endocrinology 149 1820-1828. (doi:10.1210/en.20070993)

Martin D, Epelbaum J, Bluet-Pajot MT, Prelot M, Kordon C \& Durand D 1985 Thyroidectomy abolishes pulsatile growth hormone secretion without affecting hypothalamic somatostatin. Neuroendocrinology 41 476-481. (doi:10.1159/000124222)

Nilsson O \& Baron J 2004 Fundamental limits on longitudinal bone growth: growth plate senescence and epiphyseal fusion. Trends in Endocrinology and Metabolism 15 370-374. (doi:10.1016/j.tem.2004.08.004)

Nilsson O, Marino R, De Luca F, Phillip M \& Baron J 2005a Endocrine regulation of the growth plate. Hormone Research 64 157-165. (doi:10. $1159 / 000088791)$

Nilsson O, Mitchum RD Jr, Schrier L, Ferns SP, Barnes KM, Troendle JF \& Baron J 2005b Growth plate senescence is associated with loss of DNA methylation. Journal of Endocrinology 186 241-249. (doi:10.1677/joe.1. 06016)

Nilsson O, Parker EA, Hegde A, Chau M, Barnes KM \& Baron J 2007 Gradients in bone morphogenetic protein-related gene expression across the growth plate. Journal of Endocrinology 193 75-84. (doi:10.1677/joe.1.07099)

Ogawa Y, Okada H, Heya T \& Shimamoto T 1989 Controlled release of LHRH agonist, leuprolide acetate, from microcapsules: serum drug level profiles and pharmacological effects in animals. Journal of Pharmacy and Pharmacology 41 439-444.

Pape L, Hoppe J, Becker T, Ehrich JH, Neipp M, Ahlenstiel T \& Offner G 2006 Superior long-term graft function and better growth of grafts in children receiving kidneys from paediatric compared with adult donors. Nephrology, Dialysis, Transplantation 21 2596-2600. (doi:10.1093/ndt/ gf119)

Phornphutkul C, Wu KY, Auyeung V, Chen Q \& Gruppuso PA 2008 mTOR signaling contributes to chondrocyte differentiation. Developmental Dynamics 237 702-712. (doi:10.1002/dvdy.21464)

Phornphutkul C, Lee M, Voigt C, Wu KY, Ehrlich MG, Gruppuso PA \& Chen Q 2009 The effect of rapamycin on bone growth in rabbits. Journal of Orthopaedic Research 27 1157-1161. (doi:10.1002/jor.20894)

Ringrose L \& Paro R 2004 Epigenetic regulation of cellular memory by the Polycomb and Trithorax group proteins. Annual Review of Genetics 38 413-443. (doi:10.1146/annurev.genet.38.072902.091907) 
Schenk RK \& Hunziker EB 1991 Growth plate: histophysiology, cell and matrix turnover. In Rickets, pp 63-76. Ed. FH Glorieux. New York, NY: Vevey/Raven Press.

Schrier L, Ferns SP, Barnes KM, Emons JA, Newman EI, Nilsson O \& Baron J 2006 Depletion of resting zone chondrocytes during growth plate senescence. Journal of Endocrinology 189 27-36. (doi:10.1677/joe.1.06489) Stevens DA, Hasserjian RP, Robson H, Siebler T, Shalet SM \& Williams GR 2000 Thyroid hormones regulate hypertrophic chondrocyte differentiation and expression of parathyroid hormone-related peptide and its receptor during endochondral bone formation. Journal of Bone and Mineral Research 15 2431-2442. (doi:10.1359/jbmr.2000.15.12.2431)
Walker KV \& Kember NF 1972 Cell kinetics of growth cartilage in the rat tibia. I. Measurements in young male rats. Cell and Tissue Kinetics 5 401-408. (doi:10.1111/j.1365-2184.1972.tb00378.x)

Received in final form 18 October 2010 Accepted 25 October 2010 Made available online as an Accepted Preprint 25 October 2010 